that each of these cases further substantiates the principle of electronic tautomerism or the existence of electromers in dynamic equilibrium. ${ }^{1}$

Cincinnatr, 0 .

[CONTRIbution From the Organic Chemical Laboratory of the State University OF IOWA.]

\title{
4-NITRO-5-METHYL-2-SULFOBENZOIC ACID AND SOME OF ITS DERIVATIVES.
}

By William J. Karslake and Perry A. Bond.

Received March 29, 1916.

In an earlier paper $^{2}$ we have shown that when 6-nitro-r,3-dimethylbenzene-4-sulfonic acid is oxidized by potassium permanganate in very dilute potassium hydroxide solution, three main products are formed, to wit, 6-nitro-3-methyl-4-sulfobenzoic acid, 4-nitro-5-methyl-2-sulfobenzoic acid and 6-nitro-4-sulfoisophthalic acid. Sometime later, Karslake and Huston ${ }^{3}$ made derivatives of 6-nitro-3-methyl-4-sulfobenzoic acid. The present work was undertaken to complete our knowledge of the second of these three acids.

In our previous work, 4-nitro-5-methyl-2-sulfobenzoic acid was obtained in the form of the neutral potassium salt as the first crop of crystals from the oxidized nitro-xylene sulfonic acid. Analyses of this salt were persistently low in potassium. Since then it has been found that this first crop of crystals is usually contaminated with unoxidized 6-nitro- $1,3-$ dimethylbenzene-4-sulfonic acid in the form of the potassium salt. The separation was accomplished finally by systematic fractional crystalliza. tion, the crystals being filtered off first from the fairly concentrated solu. tion at $50^{\circ}$, and then from the cold, somewhat diluted filtrate after stand.

${ }^{1}$ Any objection to the use of the conception of electronic tautomerism on the grounds that electronic isomers (electromers) have not as yet been isolated is unwarranted because the conception of tautomerism, both in its historical and practical aspects, neither involves nor demands the immediate possibility of the isolation of the tautomers. In I 885, Laar (Ber., 18, 648 (I885)), in a paper "Ueber die Möglichkeit mehrerer Strukturformeln für dieselbe chemisch Verbindung," defined tautomerism as the phenomenon of one substance doing duty for two structural isomers, but the isolation of dynamic isamers or desmotropes was not effected until 1893 , the earliest examples being acetyldibenzoylmethane, tribenzoylmethane, and mesityloxide-oxalic ester described by Claisen ( $A n n$, 277, I83 (1893); 291, 25 (1896)). Strictly speaking, the term tautomeric is now applied to substances in which only the equilibrium mixture of the structural isomers is known; the term desmotropic is employed in cases where the isomers have been shown to be capable of independent existence. (Clarke, "Organic Chemistry," p. 25I.) The existence of electromers in dynamic equilibrium has been conclusively indicated. Their isolation as electronic desmotropes has not as yet been effected. Further discussion of this phase of the subject is reserved for future papers.

2 ThIS JOURNAL, 3I, 405 (I909).

3 Ibid., 3I, 1057 (1909). 
ing several hours. The products thus obtained were recrystallized with due attention to temperature and concentration, until a complete separation was effected. About one hundred separate crystallizations were made in purifying the 235 grams of potassium salt used as a basis for this research. The influence of the impurity has very definite effects, and its removal was a necessity, as will be seen especially in the preparation of the acid chlorides. Analyses of the purified potassium salt, while slightly low, are much better than before.

Calc. for $\mathrm{C}_{8} \mathrm{H}_{5} \mathrm{O}_{7} \mathrm{NSK}_{2}: \mathrm{K}, 23.2 \mathrm{I}$. Found: $\mathrm{K}, 22.95,23.0 \mathrm{I}$.

The Neutral Lead Salt, $\mathrm{C}_{8} \mathrm{H}_{5} \mathrm{O}_{7} \mathrm{NSPb}+{ }_{3} \mathrm{H}_{2} \mathrm{O}$, was prepared by adding slightly more than the calculated amount of lead nitrate to the water solution of the potassium salt. It crystallizes quickly from moderately concentrated solution in fine crystals, which in some cases form leaf-like groups. Lead was determined by direct ignition in the crucible as the sulfate.

Calc. for $\mathrm{Pb}, 39.8 ; \mathrm{H}_{2} \mathrm{O}$, I0.38. Found: $\mathrm{Pb}, 39.45 ; \mathrm{H}_{2} \mathrm{O}$, 10.00.

The Acid Potassium Salt, $\mathrm{C}_{8} \mathrm{H}_{6} \mathrm{O}_{7} \mathrm{NSK}+\mathrm{H}_{2} \mathrm{O}$, was prepared in two ways: either by precipitating the silver from the silver potassium salt by dilute hydrochloric acid, evaporating and crystallizing; or by recrystallizing the neutral potassium salt from very concentrated hydrochloric acid. In the latter case, unless the hydrochloric acid is strong in the solution, the neutral potassium salt crystallizes out again, probably on account of its lesser solubility in water. The acid potassium salt once formed, however, can be recrystallized from water without decomposition. It comes out of the solution in beautiful, fine needles containing one molecule of water of crystallization.

Calc. for $\mathrm{K}$, I2.33; acid hydrogen, $0.3 \mathrm{I} 5 ; \mathrm{H}_{2} \mathrm{O}, 5.67$.

Found: K, I I.8I, I I.92; acid hydrogen, $0.317 ; \mathrm{H}_{2} \mathrm{O}, 5.74$.

The Free Acid, $\mathrm{C}_{8} \mathrm{H}_{7} \mathrm{O}_{7} \mathrm{NS}+{ }_{3} \mathrm{H}_{2} \mathrm{O}$, was prepared from the pure neutral silver salt by adding just a trifle more than the calculated amount of o. I $N$ hydrochloric acid to the water solution of the salt, boiling, filtering, and evaporating to a small volume. The acid crystallized from water in plate-like needles strongly rosetted and easily broken. It had only a slight yellowish tint. It was insoluble in ether, ligroin, and carbon tetrachloride. It was soluble in acetone, ethyl acetate, or glacial acetic acid. Its melting point when it was crystallized from acetone or alcohol was $94^{\circ}$. The acid was titrated with sodium hydroxide, using phenolphthalein as the indicator.

Calc. for $\mathrm{N}, 4.44$; acid hydrogen, $0.635 ; \mathrm{H}_{2} \mathrm{O}, \mathrm{I} 7.14$.

Found: $\mathrm{N}, 4.45$; acid hydrogen, $0.626 ; \mathrm{H}_{2} \mathrm{O}, \mathrm{I} 7.22$.

The Acid Chlorides.- It was formerly stated ${ }^{1}$ that the treatment of the

${ }^{1}$ Loc. cit. (Karslake and Bond). 
potassium salt of the 4-nitro-5-methyl-2-sulfobenzoic acid with phosphorus pentachloride in a flask with reflux condenser at boiling temperature produced an oily substance which solidified with difficulty, but on being dissolved in carbon tetrachloride, dried, and partly evaporated, gave two products, one melting at $133^{\circ}$, the other an oily substance for which a melting point of $93^{\circ}$ was suggested. It had been the experience of others ${ }^{1}$ that the low-melting and high-melting chlorides crystallized in a constant melting mixture which had a lower melting point than that of the low-melting chloride. The two were separated in these cases by distilling in a vacuum.

The formerly observed melting point for the high melting chloride is approximately correct, the new value adopted being $\mathrm{I} 34^{\circ}$, but now it has been definitely shown that the low melting point acid chloride melts at $83^{\circ}$, and is not involved as the prime cause of the oily mixture. In this case, as distinguished from the experience of others, no oil was produced when pure initial potassium salt was used, the two chlorides crystallizing out in absolutely distinct crystalline form. They were separated by fractional crystallization, and the symmetrical, high-melting chloride showed a tendency toward the formation of supersaturated solutions in carbon tetrachloride, the solvent used for the purification. The oily mass formerly found was, however, reproduced by mixing with the lowmelting acid chloride, a portion of the acid chloride of the unoxidized nitro-xylene sulfonic acid. Its source in that work was evidently the impurity mentioned at the beginning of this paper.

Symmetrical Acid Chloride, $\stackrel{4}{\mathrm{NO}_{2}} \cdot \stackrel{5}{\mathrm{CH}_{3}} \cdot \mathrm{C}_{6} \mathrm{H}_{2} \cdot \mathrm{SO}_{2} \mathrm{Cl} \cdot \mathrm{COCl}$. - The symmetrical acid chloride was formed in the largest amounts when the potassium salt of the acid was treated with phosphorus pentachloride in an open dish at the temperature of the steam bath, the ratio of the symmetrical to the unsymmetrical acid chloride thus formed being about three to five, and the total yield reaching $75 \%$ of the theoretical.

When the heating had caused complete liquefaction of the mass in the open dish, it was removed from the steam bath and poured into cold water. Phosphorus oxychloride and any phosphorus pentachloride remaining were dissolved in the water, and after a time the oil hardened. It was broken up with a stirring rod and ground with fresh water in a mortar. Finally the powder was let stand in more water over night, filtered, and the solid dried over sulfuric acid in a desiccator. It was next dissolved in hot carbon tetrachloride, filtered, and let stand to crystallize. If the solution was concentrated, the unsymmetrical chloride crystallized first and later there were superimposed on the crystalline mass hard, glistening, rhombohedral crystals of the symmetrical chloride. These

${ }^{1}$ Remsen, Am. Chem. J., 30, 247 (I903); List and Stein, Ber., 3I, 1648 (I898). 
were picked out by hand from the mass. In another experiment it was found that this chloride crystallized in the form of long, white needles. Once these were produced in the laboratory, the rhombohedral forms never appeared again. The needles seemed to be less soluble than the unsymmetrical acid chloride, and hence could be taken out from the carbon tetrachloride solution as the first crop of crystals. In some cases the solution was seeded to bring about the rapid production of this form.

The identity of the two crystalline forms of the symmetrical acid chloride was shown by the identical melting points, $134^{\circ}$, and also by the fact that when the rhombohedral form was dissolved in carbon tetrachloride and a trace of the needles added, the whole amount present crystallized out in the needle form.

The symmetrical acid chloride was recrystallized from carbon tetrachloride several times until the melting point was constant at $134^{\circ}$, and then analyzed.

Calc. for $\mathrm{Cl}, 23.80 ; \mathrm{N}, 4.70 ; \mathrm{S}$, 10.74. Found: $\mathrm{Cl}, 23.65,24.02 ; \mathrm{N}, 4.68,4.64 ; \mathrm{S}$, 10.72 .

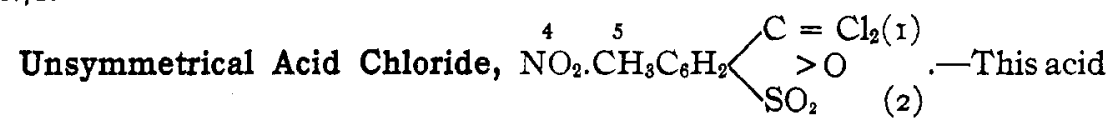
chloride constituted about five-eighths of the total yield of the acid chlorides as made by the open dish method. Its formation is apparently favored by the presence of phosphorus oxychloride, for when a reflux condenser is used in the preparation of the chlorides, and the condensed phosphorus oxychloride formed is kept in the mixture, a much larger percentage of the unsymmetrical chloride is produced, although the absolute yield seems to be smaller. Holmes ${ }^{1}$ used phosphorus oxychloride in sealed tubes for the preparation of the pure unsymmetrical acid chloride of $p$-nitro-o-sulfobenzoic acid. In this case we found it not necessary, and the shorter process was used.

The purification of the unsymmetrical acid chloride by fractional crystallization from carbon tetrachloride was not difficult. It crystallized in short, tufted, rather soft needles, yellowish in color. An attempt was made to use the method of List and Stein ${ }^{2}$ for the purification of the acid chlorides by distillation in vacuo. It was expected that the symmetrical chloride would decompose at the distillation temperature, while the unsymmetrical would distil unchanged. Distillation took place at the following temperatures and pressures:

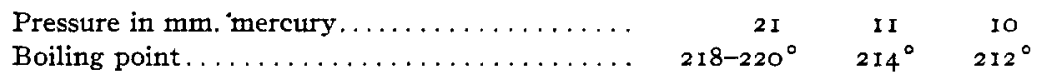

No difference was observed in the melting point of the pure $83^{\circ}$ melting point substance by the distillation.

\footnotetext{
${ }^{1}$ Am. Chem. J., 25, 204 (190I).

${ }^{2}$ Ber., 3I, 1648 (1898).
} 
The acid chloride melting at $83^{\circ}$ was analyzed, the methods used being the same as in the case of the high melting chloride.

Calc. for $\mathrm{Cl}, 23.80 ; \mathrm{N}, 4.70 ; \mathrm{S}, 10.74$.

Found: $\mathrm{Cl}, 23.3,23.4,23.46 ; \mathrm{N}, 4.82,4.79 ; \mathrm{S}, 10.77$.

\section{Derivatives Obtained by the Action of Ammonia on the Acid Chlorides.}

A. From the Symmetrical Acid Chloride.-A suitable amount of the acid chloride was placed in an open beaker and treated with concentrated ammonia. Apparently there was no action in the cold, but on raising the temperature slowly to boiling, so as to keep the concentration of the ammonia as high as possible, the acid chloride dissolved. When the excess of ammonia was evaporated off and the residue was cooled somewhat, the ammonium salt of the imide crystallized out as small glistening cubes. These were recrystallized from water several times. They then gave a melting point, with decomposition, of $310-320^{\circ}$. No other substance was recovered in the preparation except the ammonium chloride formed in the reaction. The symmetrical acid chloride is only slightly acted upon in nonaqueous solutions by ammonia.

Calc. for $\mathrm{C}_{8} \mathrm{H}_{8} \mathrm{O}_{5} \mathrm{~N}_{8} \mathrm{~S}$ : Total N, 16.21; Ammon. N, 5.40; S, 12.35.

Found: Total N, I6.20, 16.18; Ammon. N, 5.58; S, I2.38.

The Imide, $\stackrel{4}{\mathrm{NO}_{2}} \cdot \stackrel{5}{\mathrm{CH}_{3}} \cdot \mathrm{C}_{6} \mathrm{H}_{2}\left\langle\stackrel{+-2}{\mathrm{CO}}{ }_{\mathrm{SO}_{2}}\right\rangle \mathrm{NH}$.-When the cold mother liquor from the crystallization of the ammonium salt of the imide is made acid with hydrochloric acid, the imide separates almost quantitatively from the solution. It is colorless, and the crystals as viewed under the microscope are platelike needles. The insolubility of the precipitate makes it unnecessary to purify except by washing with water until free from chlorides. The imide is bitter tasting. It is readily soluble in alkali and forms salts with various metals by which it may be characterized more completely. Its melting point is $2 \mathrm{I} 3.5^{\circ}$.

Calc. for $\mathrm{N}, \mathrm{x} 1.57$; S, 13.22. Found: $\mathrm{N}, \mathrm{x1} .77, x \mathrm{x} .68 ; \mathrm{S}, 13.25$.

The silver salt of the imide, $\mathrm{C}_{8} \mathrm{H}_{5} \mathrm{O}_{5} \mathrm{SN}_{2} \mathrm{Ag}$, was obtained by adding a solution of silver nitrate to a hot aqueous solution of the imide. It separates in platelike needles and is less soluble than the silver salt of the cyan-acid to be described later.

Calc. for $\mathrm{Ag}, 30.92$. Found: $\mathrm{Ag}, 30.87$.

The potassium salt of the imide was obtained by adding an excess of a concentrated potassium carbonate solution to an aqueous solution of the ammonium salt. It crystallizes in plates, and has a weak bitter taste.

Calc. for $\mathrm{K}, 13.96$. Found: $\mathrm{K}, \mathrm{I} 3.9 \mathrm{I}$.

$B$. From the Unsymmetrical Chloride.-If the acid chloride melting at $83^{\circ}$ is treated with cold concentrated ammonia, an action takes place 
which, while beginning slowly, at last goes on rapidly with the evolution of heat. The behavior in this respect is characteristically different from that of the symmetrical chloride. In making the ammonia derivative in practice, the ammonia was diluted with three parts of water to lessen the action. If the ammonia is evaporated off and the remaining liquid is concentrated and cooled, crystals form in nodules. These crystals are not as sharply defined as those of the corresponding ammonium salt of the imide; the nodules appear to be made up of compact masses of radiating crystals which branch continually into fanlike shapes. When these nodules are crushed, it is impossible to find any characteristic crystalline form. Recrystallized from alcohol, however, the crystals were not bunched but seemed to be laminated plates almost cubical in form, but with no smooth surfaces. The yield of the product is good. In no case was there any indication of the ammonium salt of the imide. The salt is more soluble than the ammonium salt of the imide. Its melting point is $310-3 I^{\circ}$, with decomposition. It is the ammonium salt of $5^{-}$ nitro-4-methyl-2-cyanbenzenesulfonic acid. It has a very bitter taste. The free acid was not prepared.

Calc. for $\mathrm{C}_{8} \mathrm{H}_{8} \mathrm{O}_{5} \mathrm{~N}_{3} \mathrm{~S}: \mathrm{N}, 16.2 \mathrm{I} ; \mathrm{S}, 12.35$. Found: N, $16 . \mathrm{II}, 15.95 ; \mathrm{S}, \mathrm{I2.31}, 12.24$.

The silver salt, $\mathrm{C}_{8} \mathrm{H}_{5} \mathrm{O}_{5} \mathrm{SN}_{2} \mathrm{Ag}$, was prepared by adding a silver nitrate solution to a hot dilute aqueous solution of the ammonium salt. It forms needles and contains no water of crystallization.

Calc. for $\mathrm{Ag}, 30.92$. Found: $30.8 \mathrm{I}$.

The potassium salt, $\mathrm{C}_{8} \mathrm{H}_{5} \mathrm{O}_{5} \mathrm{SN}_{2} \mathrm{~K}$, made from the ammonium salt and potassium carbonate, separates in plates with no water of crystallization. It has a bitter taste.

Calc. for K, 13.96. Found: 14.03.

\section{Derivatives Obtained by the Action of Aniline on the Acid Chlorides.}

$A$. From the Symmetrical Chloride.--The formation of the anilides of the acid chlorides by previous experimenters in this field has been carried out in three ways: first, by the treatment of the solid acid chloride with aniline; second, by the treatment of the acid chloride with aniline in ether or chloroform solution; and third, by the action of aniline on the acid chloride in the presence of water. The first method has been found to be unsatisfactory. In general, the third method has been chosen by Remsen and his students as it seemed to give the most constant results. In this work, however, it has been found that by using carbon tetrachloride as the solvent the products were of the same nature as those obtained by the aqueous method. The process is shorter and the substances are easily separated from one another.

From the symmetrical acid chloride two products were obtained. One of these, which is insoluble in cold, reasonably dilute sodium hydroxide, 
is known as the anil. It resembles the imide in many respects, but does not form salts as it contains no imido hydrogen. The other product is the symmetrical dianilide. ${ }^{1}$

The Anil, $\stackrel{4}{\mathrm{NO}_{2}} \cdot \stackrel{5}{\mathrm{CH}_{3}} \cdot \mathrm{C}_{6} \mathrm{H}_{2} \underset{\mathrm{SO}_{2}}{>} \underset{\mathrm{N} . \mathrm{C}_{6} \mathrm{H}_{5}}{\mathrm{C}}$. - - One gram of the symmetrical acid chloride was dissolved in about $50 \mathrm{cc}$. of carbon tetrachloride at boiling temperature. While this was hot there was added aniline in excess of the amount calculated for the dianilide. This aniline also was dissolved in carbon tetrachloride, and the mixture was warmed for an hour. There was only a slight precipitate at the end of that time, hence the carbon tetrachloride was evaporated and the residue treated with dilute hydrochloric acid. After kneading the residue, which at this stage was oily in nature, for several minutes, the mass solidified. It was then broken up into fine fragments and was allowed to stand overnight in order that the excess aniline might all dissolve as the hydrochloride. The residue was filtered, ground in a mortar with hydrochloric acid, filtered and washed with water to remove the excess of acid. It was next treated with cold, dilute potassium hydroxide. The solution became bright yellow, and a dirty white precipitate was left in an amount small as compared with the bulk of the original precipitate. This insoltuble material, which was the anil, was washed with water, dried, and recrystallized from alcohol. The yield of crude anil was $0.27 \mathrm{~g}$.

The anil is very insoluble in alcohol except close to the boiling point. About one hundred $\mathrm{cc}$. of alcohol were required to recrystallize the anil as above. When pure, it is nearly white, and crystallizes in needles which have a melting point of $202-203^{\circ}$.

Calc. for $N, 8.81$. Found: N, 9.00 .

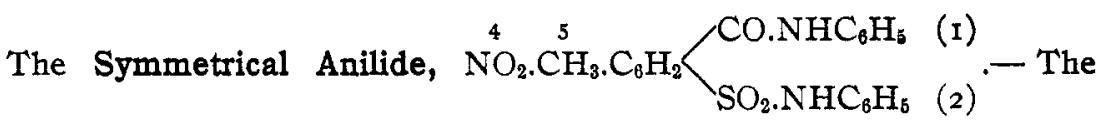
sodium hydroxide solution filtered from the anil was acidified with hydrochloric acid. A white, curdy precipitate at once came down and was filtered off and washed free from acid. It was then redissolved and reprecipitated for further purification. Finally, it was washed, dried, and recrystallized from $75 \%$ alcohol. It formed a fine, cottony mass of soft needles with a melting point of $195^{\circ}$. The yield of the anilide was $0.98 \mathrm{~g}$. The substance was identified in this case not by analysis but by its exact correspondence with the dianilide formed under like conditions as a derivative of the unsymmetrical acid chloride. Mixtures of the two preparations showed the same melting point as that of either of the pure specimens.

${ }^{1}$ See Remsen and Kohler, $A m$. Chem. J., 17, 338 (1895). 
List and Stein ${ }^{1}$ state that there are three products formed by the action of aniline on the symmetrical chloride, but there was no indication of a third compound here, and a consideration of the yields obtained will show that no very large amount of the material is unaccounted for.

$B$. From the Unsymmetrical Chloride.-The unsymmetrical chloride was dissolved in carbon tetrachloride and to the hot solution was added a carbon tetrachloride solution of aniline so long as a white precipitate continued to form. Hydrochloric acid vapor was evolved copiously, and aniline hydrochloride apparently did not form to any great extent. The precipitate thus formed, on being stirred, gathered into a gummy mass which hardened as the solution cooled. The carbon tetrachloride solution was evaporated to dryness and the whole solid residue ground with hydrochloric acid in a mortar and then allowed to stand to remove soluble products. After some hours it was filtered and washed with a considerable amount of water. The insoluble precipitate was treated with dilute sodium hydroxide in which it was almost completely soluble. Only a faint cloudiness remained in the solution, hence but the merest traces of anil could have been formed. This corresponds with the observation by Holmes ${ }^{2}$ that no anil was produced from the unsymmetrical chloride of 0 -sulfobenzoic acid. From this solution the anilide was precipitated by hydrochloric acid, washed, dried and recrystallized from alcohol. It was found, however, that a large portion of the precipitated material would not dissolve in hot alcohol. The insoluble portion proved to be the unsymmetrical anilide described later. The portion which dissolved gave needles which melted at $195^{\circ}$, and were the symmetrical anilide. It is tasteless, and identical with the anilide formed from the symmetrical acid chloride.

Calc. for $\mathrm{N}$, 10.22; S, 7.78. Found: $\mathrm{N}, 10.15$, 10.06; S, 7.91 .

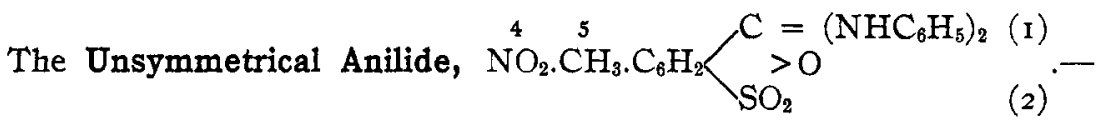

The substance insoluble in alcohol was purified by dissolving in hot $75 \%$ alcohol with the aid of sodium hydroxide added drop by drop until the precipitate had disappeared. To this solution acid was added, and the precipitate which formed after a delay of some seconds was nicely crystalline. The crystals were very fine. The melting point seemed to be at $342^{\circ}$; but fusion did not occur until after the product was quite black with heating.

The unsymmetrical anilide is insoluble in most ordinary solvents and this is a general characteristic of such substances. Carbon tetrachloride, alcohol and benzene have little, if any, action on it.

${ }^{1}$ Loc. cit.

2 Am. Chem. J., 30, 275 (1903). 
Calc. for $\mathrm{N}, 10.22 ; \mathrm{S}, 7.78$. Found: $\mathrm{N}, 10.02 ; \mathrm{S}, 7.77,7.69$.

A third substance is produced by the action of aniline on the unsymmetrical chloride. It is soluble in water and may be recovered from the various washings of the original dianilide by evaporation. It crystallizes in needles. It is suspected that this body is the anilido acid, but it is difficult to purify and no satisfactory analyses were obtained of it. While the total yields of the two anilides obtained from the unsymmetrical acid chloride were almost equal in amount, the weight of the third substance is considerably less than either.

\section{Transformations of the Aniline Derivatives.}

When either the symmetrical or unsymmetrical anilide is treated with phosphorus oxychloride, at boiling temperature, for about onehalf hour, a yellow colored solution is produced. If the excess of phosphorus oxychloride is distilled off with care, an air blast being used to help the process, a thick, brownish oil is left, which after being cooled and treated with water to remove phosphoric acid, becomes a bright yellow powder. This powder is the dianil, $\mathrm{NO}_{2}^{4} \cdot \mathrm{CH}_{3} \cdot \mathrm{C}_{6} \mathrm{H}_{2}<\underset{\mathrm{SO}_{2}}{>\mathrm{NC}_{6} \mathrm{H}_{5}}$ (2) It crystallizes from dilute acetone in short, bright yellow, hexagonal needles. It is soluble in benzene and alcohol, and melts at $188^{\circ}$.

Calc. for N, 10.69; S, 8.14. Found: N, 10.76; S, 8.10.

When the dianil ${ }^{1}$ is boiled with concentrated hydrochloric acid it goes over into the colorless anil without dissolving, and the yellow color slowly disappears during the process. The melting point of the colorless solid, $202^{\circ}$, shows it to be the anil.

If the dianil is boiled for some time with glacial acetic acid until the solution, which is vivid yellow at first, becomes colorless, and the solution is allowed to stand, the unsymmetrical anilide crystallizes out. When washed with water and dried it has a melting point of approximately $34^{\circ}$, as described previously.

If the anil is warmed with aniline for some hours, and the excess of aniline distilled off with steam, the residue when recrystallized from alcohol, proves to be the fusible or symmetrical anilide with a melting point of $195^{\circ}$.

\section{Derivatives Obtained by the Action of Alcohol on the Acid Chlorides.}

In all of the experiments with the alcoholic derivatives of the two acid chlorides, the final products are the same, the only difference being the formation of the ester of the sulfone chloride as an intermediate product in the action of the alcohol on the unsymmetrical acid chloride. ${ }^{2}$

${ }^{1}$ See Blanchard, Am. Chem. J., 30, 485 (1903); Henderson, Ibid., 25, 1 (I9or).

${ }^{2}$ See Kastle, Am. Chem. J., II, I8I (I889); Sohon, Ibid., 20, 260 (I898); Henderson, Ibid., 25, 8 (1901); Chambers, Ibid., 30, 387 (1903). 


\section{Methyl Ester Sulfone Chloride, $\stackrel{4}{\mathrm{NO}_{2}} \stackrel{5}{\mathrm{CH}_{3}} \cdot \mathrm{C}_{6} \mathrm{H}_{2} \cdot \mathrm{COOCH}_{3} \cdot \mathrm{SO}_{2} \mathrm{Cl}$ -} This substance was prepared from the unsymmetrical acid chloride by dissolving in hot methyl alcohol. The warming was kept up only long enough to obtain solution. When the solution was cooled, the sulfone chloride ester separated in long, platelike crystals which were recrystallized from ligroin. It is very soluble in benzene, alcohol, acetone, ether and chloroform. After standing overnight in a desiccator, the product, which has a melting point of $\mathrm{IOI}^{\circ}$, was analyzed.

Calc. for $\mathrm{N}, 4.77 ; \mathrm{S}, 10.92 ; \mathrm{Cl}, 12.08$.

Found: N, 4.66, 4.6I; S, 10.75, I1.02; Cl, 12.09, 12.01.

The ethyl ester sulfone chloride was also prepared and analyzed. It melts at $72^{\circ}$.

\section{Methyl Benzoic Ester of the Sulfonic Acid, $\stackrel{4}{\mathrm{NO}_{2}} \stackrel{5}{\mathrm{CH}_{3}} \cdot \mathrm{C}_{6} \mathrm{H}_{2} . \mathrm{COOCH}_{3}$. 2}

$\mathrm{SO}_{2} \mathrm{OH}$.- The methyl ester acid was prepared by boiling either acid chloride with methyl alcohol for some time and evaporating to a syrup. On cooling, it slowly formed crystals which, on account of their extreme solubility, were not purified. The identification was by means of the barium salt, which could be made easily. The silver salt, which was very soluble, was made but not analyzed. It was used in the preparation of the dimethyl ester. The barium salt contains no water of crystallization, and gave the following result upon analysis:

$$
\text { Calc. for } \mathrm{Ba}, 20.04 \text {. Found: } \mathrm{Ba}, 20.05 \text {. }
$$

The ethyl benzoic ester was also prepared.

The , Dimethyl Ester, $\stackrel{4}{\mathrm{NO}_{2}} \cdot \stackrel{5}{\mathrm{CH}_{3}} \cdot \mathrm{C}_{6} \mathrm{H}_{2} \cdot \mathrm{COOCH}_{3} \cdot \mathrm{SO}_{2}^{2} \mathrm{OCH}_{3}$. - The dimethyl ester cannot be made by the direct action of the acid chlorides on alcohol. Neither does the action of sodium methylate, ${ }^{1}$ either dry or in absolute alcohol, upon an absolute ether solution of the acid chloride prove of much value. The best method was that of treating the silver salt of the methyl ester acid with methyl iodide in a pressure bottle as recommended by Kastle. ${ }^{2}$ After heating the mixture some time at $100^{\circ}$ the excess of methyl iodide is evaporated off and the residue extracted with absolute ether. The product obtained by evaporating the ether and recrystallizing from absolute methyl alcohol melted at $94.5^{\circ}$. It is insoluble in water, has a neutral reaction, and contains no halogen.

Calc. for N, 4.85. Found: N, 4.98.

\section{Derivatives Obtained by the Action of Phenol on the Acid Chlorides.}

The action of phenol upon the acid chlorides is of the same general nature as that given by the alcohols. Both acid chlorides seem to give

${ }^{1}$ Cobb, Am. Chem. J., 35, 488 (1908).

${ }^{2}$ Loc. cit. 
practically the same derivatives, ${ }^{1}$ the relative amounts of which depend upon the temperature. At the same time there is usually formed a watersoluble red substance, probably a phthalein, which has not been investigated.

The Diphenyl Ester, $\stackrel{4}{\mathrm{NO}_{2}} \cdot \stackrel{5}{\mathrm{CH}_{3}} \cdot \mathrm{C}_{6} \mathrm{H}_{2} \cdot \mathrm{COOOC}_{6} \mathrm{H}_{6} \cdot \mathrm{SO}_{2} \mathrm{OC}_{6} \mathrm{H}_{6}$. - Three methods were used in preparing the diphenyl ester. First, the acid chloride was warmed with phenol. The production of phthalein was so marked that this method was used only for a trial preparation. The di-ester appears to be the only product outside of the phthalein.

Second, the Schotten-Baumann method was tried. The acid chloride was dissolved by gentle warming in a slight excess of phenol and dilute potassium hydroxide solution. The yellow oil which first formed soon solidified and was recrystallized from organic solvents. The amount of phthalein formed was small.

The third method consisted of treating the acid chloride with a mixture of pyridine and phenol. Two grams of phenol and an equal amount of pyridine were mixed in a small beaker and the powdered acid chloride added.

When the acid chloride had dissolved in the pyridine phenol solution the liquid was allowed to stand at room temperature for the completion of the reaction. Alcohol was then added and warmed until all the compound was in solution. As the liquid cooled, crystals formed as colorless, flat needles somewhat resembling those of the ethyl benzoic ester of the sulfone chloride. These contained no chlorine, however, and were the diphenyl ester. The melting point of the crude product is, according to our experience, always $115^{\circ}$ or $116^{\circ}$, but by recrystallization from alcohol, benzene, alcohol, glacial acetic acid, and alcohol in the order named, the melting point becomes constant at $123^{\circ}$. The products which were obtained separately from the symmetrical and unsymmetrical acid chlorides are identical in this respect as in all others. No sulfone chloride was formed by any of these three methods, a fact which was due in all probability to the temperature employed.

Calc. for $\mathrm{N}, 3.39 ; \mathrm{S}, 7.75$. Found: $\mathrm{N}, 3.42,3.47 ; \mathrm{S}, 7.74,7.86$.

\section{Summary.}

I. Two acid chlorides, the symmetrical with a melting point of $134^{\circ}$, the unsymmetrical with a melting point of $83^{\circ}$, have been prepared. These compounds were peculiar only in that they could be separated by fractional crystallization from solution, and that when pure, they did not tend in the least to form constant melting-point mixtures. Two crystalline forms of the symmetrical acid chloride were isolated, but their differences were not investigated closely.

${ }^{1}$ Humphreys, Am. Chem. J., 30, 292 (1903); Chambers, Ibid., 30, 374 (1903). 
2. The derivative obtained by the action of ammonia upon the symmetrical acid chloride was the ammonium salt of the imide. From this the imide itself was prepared by the action of dilute hydrochloric acid. The silver salt and the potassium salt of the imide also were made and analyzed.

3. The derivative obtained by the action of ammonia on the unsymmetrical acid chloride was the ammonium salt of the cyan acid; no imide was found. The silver and potassium salts of the cyan acid also were made and analyzed.

4. The derivatives obtained by the action of aniline on the symmetrical acid chloride were the symmetrical dianilide and the anil.

5. The derivatives obtained by the action of aniline on the unsymmetrical acid chloride were the symmetrical dianilide and the unsymmetrical dianilide.

6. By the action of phosphorus oxychloride, each of the two dianilides was transformed into the dianil. This in turn when heated with concentrated hydrochloric acid gave the anil or, heated with glacial acetic acid it gave the unsymmetrical dianilide.

7. The derivative obtained by the action of the alcohols on the symmetrical acid chloride was the benzoic ester of the sulfonic acid.

8. The derivatives obtained by the action of alcohols on the unsymmetrical acid chloride were the benzoic ester of the sulfone chloride, and the benzoic ester of the sulfonic acid.

9. The derivative obtained by the action of phenol upon either of the acid chlorides was always the diphenyl ester. No particular effort was made to prepare the phenyl sulfone chloride. When pyridine was used in the reaction, a second substance was formed which may be the pyridine salt of the phenyl benzoic ester acid. Its constitution was not established.

Io. By the action of methyl iodide on the silver salt of the methyl benzoic ester acid, the dimethyl ester was formed.

Iowa CIIY, IOWA.

[CONTRIBUTION FROM THE CHEMICAL LABORATORIES OF ColuMBIA UNIVERSITY.] THE SYNTHESIS OF $p$-CYMENE 2-MONOCARBOXYLIC ACID AND OF p-CYMENE 3-MONOCARBOXYLIC ACID, TOGETHER WITH CERTAIN OF THEIR DERIVATIVES.

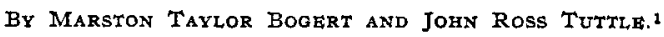

Received May 12, 1916.

The acids which are the subject of the present article are depicted by the following structural formulas:

1 The experimental work upon which this paper is based was carried out by Mr. Tuttle in partial fulfilment of the requirements for the degree of Doctor of Philosophy under the Faculty of Pure Science of Columbia University. 